\title{
Advantages of ocular regeneration research
}

It is my pleasure to serve as the guest editor for this special eye series of Annals of Translational Medicine entitled "Novel Tools and Therapeutics in Eye Regeneration." This special series is very timely since there has been a recent increase in the momentum of translational research in ocular regeneration. This increase stems partially from the success of ocular gene therapy. The US Food and Drug Administration (FDA) approval of voretigene neparvovec-rzyl (Luxturna; Spark Therapeutics) in January 2018 to treat inherited retinal diseases associated with mutations in the gene RPE65 is the first gene therapy to be approved by the FDA for clinical use. Its approval was based on very compelling positive results of the clinical trials showing improvement in visual function in individuals with previously untreatable progressive severe vision loss (1).

Conducting translational regenerative research in the eye has many advantages which likely contributed to the success of gene therapy for ocular use. The most obvious is the optically clear media of the eye that makes vision possible. The clear ocular media also allows direct in vivo visualization and examination of the various tissues in the eye, including the cornea, retina and optic nerve head in live humans and animals. The clear ocular media also allow various in vivo optical imaging tools to be developed for clinical and preclinical application. Sher et al. summarized in this series the various imaging tools that have been used for clinical and preclinical work on retinal regeneration (2). It provides a nice background on the topic. These imaging tools allow non-invasive in vivo histologic analysis of ocular tissue such that morphologic changes associated with various ocular disorders and the effects of therapy can be appreciated in vivo. Ultrahigh resolution imaging possible using adaptive optics and optical coherence tomography (OCT) can result in analysis of the retina at near cellular level. Novel development of these ultrahigh resolution in vivo imaging tools has led to the ability to conduct functional testing of the retina at subcellular level. This is exemplified in the optoretinogram discussed by Dr. Jonnal in this series (3). Optoretinogram is an in vivo retinal imaging method using adaptive optics and OCT to evaluate subcellular changes within the photoreceptor cells associated with change in photoreceptor function. This novel technology could be the most sensitive retinal functional testing tool to date.

Another advantage of conducting translational research for eye disease is the availability of well-established animal models of common ocular conditions associated with vision loss. In this series, Park et al. provide us with a comprehensive overview of animal models of corneal endothelial dysfunction that can be used for development of novel therapies (4). The cornea is the most anterior, superficial tissue in the eye. Optical clarity of the cornea is important for preserving vision. Corneal endothelial diseases affect corneal clarity and are major causes of vision loss. For the retina, dysfunction resulting from retinal ischemia or degeneration results in profound vision loss which is often irreversible. This series includes a review of animal models of diabetic retinopathy, a leading retinal vascular cause of vision loss in adults. As summarized by Quiroz and Yazdanyar, none of the animal models completely simulate human disease, but various aspects of this retinopathy can be studies using these models (5). In this series, one of the more commonly used diabetic retinopathy murine model i.e., streptozocin-induced diabetic retinopathy model, is used by Cheung et al. to show that intravitreal injection of human CD34+ bone marrow stem cells result in preservation of the retinal vessels (6). The findings are important since a phase I/II clinical trial is currently underway exploring the safety and efficacy of intravitreal injection of autologous CD34+ stem cells for vision loss associated with retinal vein occlusion, another leading retinal vascular cause of vision loss (www.clinicaltrials.gov).

Various animal models also exist for retinal degeneration, and new models continue to be developed and characterized. In this series, Salpeter et al. report on a novel murine model of retinal degeneration associated with neuronal ceroid lipofuscinosis type 8 and compares the retinal changes to that noted in humans with the same condition (7). A more commonly used model of retinal degeneration, Royal College of Surgeon (RCS) rat, is characterized by slowly progressive diffuse retinal degeneration which is hereditary and analogous to retinitis pigmentosa, a diffuse retinal degenerative condition seen in humans. In this series, Park et al. from Seoul National University collaborated with our researchers at University of California Davis to show efficacy of intravitreal and subretinal injection of CD34+ stem cells from bone marrow in preserving retinal function in a rat model of diffuse retinal degeneration (8). The results show that the regenerative effects of CD34+ stem cell is not limited to retinal vascular disease as demonstrated by Cheung et al. (6).

Disorders of the optic nerve, such as glaucoma, are also leading causes of vision loss and blindness that cannot be treated at the current time. In this series, Fague $e t$ al. provides an overview of animal models optic nerve damage and degeneration 
which can be used to develop regenerative therapy for optic nerve disorders (9). Such regenerative therapies that limit or reverse optic nerve degeneration can have high impact on vision in millions of people. The technology may also pave way to developing novel regenerative therapies in non-ocular neuronal tissue.

Another major advantage of eye research for developing regenerative therapy is the small and enclosed nature of the eye. This allows local administration of novel therapies to be conducted easily and with potential maximum therapeutic effect. Intravitreal injections of drugs are commonly administered in the clinic. Commonly injected drugs are those that inhibit vascular endothelial growth factor (VEGF), a growth factor mediating angiogenesis associated with neovascular complications of macular degeneration and diabetic retinopathy. These drugs usually need to be reinjected, often indefinitely. In this series, Chung et al. provide a review of a gene therapy approach to inhibit VEGF in the eye that is being explored in preclinical and early phase clinical trial (10). Long-term intraocular inhibition of VEGF via gene therapy could limit the number of treatments needed for patients with macular degeneration and diabetic retinopathy.

Intraocular administration of various novel regenerative therapies, including various gene therapies and stem cell therapies, are being explored in preclinical and early phase clinical trials for retinal regeneration. The successful FDA approval of Voretigene neparvovec-rzyl as gene therapy for RPE65 related retinal degeneration has heralded the development of gene therapies for other inherited retinal disorders. In this series, Nuzbrokh et al., provide a comprehensive up to date review of the gene therapy research for inherited retinal disorders, including multiple clinical trials that are on-going or recently completed (11). Most gene therapies, including the FDA approved gene therapy, Voretigene neparvovec-rzyl, is commonly administered subretinal in the operating room (1). However, intravitreal and suprachoroidal delivery options also are being explored. Similarly, clinical trials have started exploring intraocular administration of stem cells for retinal regeneration. In addition, to intravitreal injection of autologous CD34+ stem cells, intravitreal and subretinal injection of fetal and pluripotent stem cells are being explored in clinical trials (12). An up-to-date review of clinical trials exploring pluripotent stem cells is provided by Ahmed et al. in this series (13).

Finally, it is important to note that the changes in the eye can often be a manifestation of systemic disease. In this series, Smit-McBride and Morse provide a review of research being done on microRNA in diabetic mellitus and associated retinopathy (14). Specific microRNAs have been associated with diabetic retinopathy and could be used as biomarkers or future therapeutic targets.

This special eye series includes review and original articles of novel tools and therapies being developed for ocular regeneration. This area of research is very broad and cannot be completely covered in this single series. The goal of this eye series is to provide some overview of the latest research being done in the area of ocular regeneration in order to highlight the broad potential in this field. As a practicing ophthalmologist specializing in disorders of the retina, I have a personal interest in research being conducted for retinal disorders that blind many of my patients. Thus, the series may appear a bit skewed towards retinal research. However, this possible bias may be justified by the fact that leading causes of untreatable blindness currently are conditions affecting the retina or retinal ganglion cells that constitute the optic nerve.

In closing, I would like to thank my many colleagues who contributed to this research series. Despite the disruption of professional and personal daily life by the unanticipated emergence of the COVID-19 pandemic during the past year, each contributor did an outstanding job covering the various topics. This eye series would not have been possible without each of their contributions.

\section{Acknowledgments}

Funding: SSP is supported in part by BA \& AM Roth, MD Endowed Chair of Discovery, Education and Patient Care in Visual Science from the University of California Davis.

\section{Footnote}

Provenance and Peer Review: This article was commissioned by the editorial office, Annals of Translational Medicine for the series "Novel Tools and Therapies for Ocular Regeneration". The article did not undergo external peer review. 
Conflicts of Interest: The author has completed the ICMJE uniform disclosure form (available at https://dx.doi.org/10.21037/ atm-21-1793). The series "Novel Tools and Therapies for Ocular Regeneration" was commissioned by the editorial office without any funding or sponsorship. SSP served as the unpaid Guest Editor of the series and serves as an unpaid editorial board member of Annals of Translational Medicine from Oct 2019 to Sep 2021. SSP is supported by the BA \& AM Roth, MD Endowed Chair of Discovery, Education and Patient Care in Visual Science at the University of California Davis. SSP has received grant support from the National Eye Institute, Roche/Novartis and Cures within Reach. SSP has also received honoraria from National Eye Institute, US Department of Defense and George Washington University for grant review and lecture. The author has no other conflicts of interest to declare.

Ethical Statement: The author is accountable for all aspects of the work in ensuring that questions related to the accuracy or integrity of any part of the work are appropriately investigated and resolved.

Open Access Statement: This is an Open Access article distributed in accordance with the Creative Commons AttributionNonCommercial-NoDerivs 4.0 International License (CC BY-NC-ND 4.0), which permits the non-commercial replication and distribution of the article with the strict proviso that no changes or edits are made and the original work is properly cited (including links to both the formal publication through the relevant DOI and the license). See: https://creativecommons.org/licenses/by-nc$\mathrm{nd} / 4.0 \%$.

\section{References}

1. Russell S, Bennett J, Wellman JA, et al. Efficacy and safety of voretigene neparvovec (AAV2-hRPE65v2) in patients with RPE65-mediated inherited retinal dystrophy: a randomised, controlled, open-label, phase 3 trial. Lancet 2017;390:849-60.

2. Sher I, Moverman D, Ketter-Katz H, et al. In vivo retinal imaging in translational regenerative research. Ann Transl Med 2020;8:1096.

3. Jonnal RS. Toward a clinical optoretinogram: a review of noninvasive, optical tests of retinal neural function. Ann Transl Med 2021;9:1270.

4. Park S, Leonard BC, Raghunathan VK, et al. Animal models of corneal endothelial dysfunction to facilitate development of novel therapies. Ann Transl Med 2021;9:1271.

5. Quiroz J, Yazdanyar A. Animal models of diabetic retinopathy. Ann Transl Med 2021;9:1272.

6. Cheung KW, Yazdanyar A, Dolf C, et al. Analysis of the retinal capillary plexus layers in a murine model of diabetic retinopathy: effect of intravitreal injection of human CD34+ bone marrow stem cells. Ann Transl Med 2021;9:1273.

7. Salpeter EM, Leonard BC, Lopez AJ, et al. Retinal degeneration in mice and humans with neuronal ceroid lipofuscinosis type 8 . Ann Transl Med 2021;9:1274.

8. Park UC, Park SS, Kim BH, et al. Subretinal versus intravitreal administration of human CD34+ bone marrow-derived stem cells in a rat model of inherited retinal degeneration. Ann Transl Med 2021;9:1275.

9. Fague L, Liu YA, Marsh-Armstrong N. The basic science of optic nerve regeneration. Ann Transl Med 2021;9:1276.

10. Chung SH, Frick SL, Yiu G. Tageting vascular endothelial growth factor using retinal gene therapy. Ann Transl Med 2021;9:1277.

11. Nuzbrokh Y, Ragi SD, Tsang SH. Gene therapy for inherited retinal diseases. Ann Transl Med 2021;9:1278.

12. Singh MS, Park SS, Albini TA, et al. Retinal stem cell transplantation: Balancing safety and potential. Prog Retin Eye Res 2020;75:100779.

13. Ahmed I, Johnson RJ, Singh MS. Pluripotent stem cell therapy for retinal diseases. Ann Transl Med 2021;9:1279.

14. Smit-McBride Z, Morse LS. MicroRNA and diabetic retinopathy—biomarkers and novel therapeutics. Ann Transl Med 2021;9:1280. 


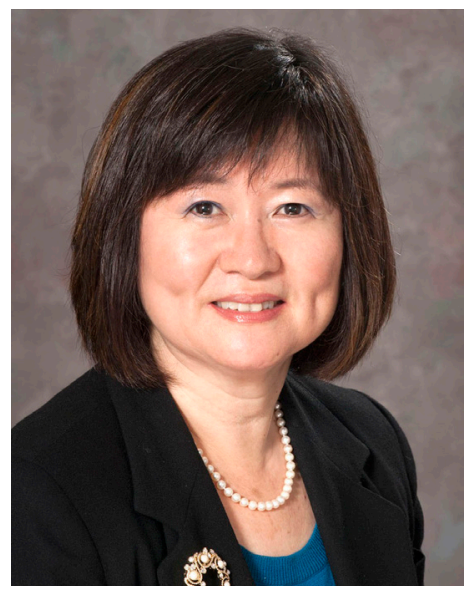

Susanna S. Park

Susanna S. Park Department of Ophthalmology \& Vision Science, University of California-Davis Eye Center, Sacramento, CA, USA. (Email: sscpark@ucdavis.edu) Submitted Apr 11, 2021. Accepted for publication Apr 29, 2021. doi: $10.21037 / \mathrm{atm}-21-1793$

View this article at: https://dx.doi.org/10.21037/atm-21-1793

Cite this article as: Park SS. Advantages of ocular regeneration research. Ann Transl Med 2021;9(15):1269. doi: 10.21037/atm-211793 